\title{
Géneros de Theaceae: aspectos taxonómicos
}

\section{Y NOMENCLATURALES}

\author{
ISOLDA LUNA ${ }^{1}$ y JOSÉ LUIS VILLASEÑOR ${ }^{2}$ \\ ' Facultad de Ciencias, Universidad Nacional Autónoma de México, Apartado postal 70-399, \\ Coyoacán, 04510 México, D. F., México. \\ ${ }^{2}$ Instituto de Biología, Departamento de Botánica. Universidad Nacional Autónoma de México, \\ Apartado postal 70-367, Coyoacán, 04510 México, D. F., México.
}

\begin{abstract}
Resumen: La familia Theaceae se distribuye en las áreas tropicales y subtropicales del mundo. Su principal centro de riqueza y diversificación se encuentra en Asia, donde están la mayoría de los géneros, entre los que se cuentan los más plesiotípicos y varios de los más derivados. Como se circunscribe en este trabajo, la familia está compuesta por 20 géneros, definidos con base en al menos una apomorfia compartida por todas sus especies. El reconocimiento de los géneros de Theaceae se efectuó mediante un examen exhaustivo de la literatura y la verificación rigurosa de numerosos caracteres considerados como de gran importancia taxonómica para su circunscripción, a partir del estudio de ejemplares depositados en algunos de los principales herbarios del mundo que contienen material de esta familia, especialmente tipos. Tomando en cuenta una hipótesis filogenética bosquejada en un trabajo en desarrollo y la síntesis de las delimitaciones genéricas para Theaceae, se determinó el nombre correcto y la sinonimia para cada uno de los géneros reconocidos.

Palabras clave: Theaceae, taxonomía, géneros.

Abstract: The family Theaceae is distributed in the tropical and subtropical regions of the world. Its main center of richness and diversification is found in Asia where most of the genera are found, including the most plesiotypic as well as several more derived. As recognized in this work, the family is composed by 20 genera, all defined by sharing all the species at least one sinapomorphy. Recognizing of the genera was the result of an exhaustive revision of literature, the rigorous verification of numerous characters of taxonomic importance by studying herbarium specimens hold in several herbaria that include good material of this family, especially types. Based on a phylogenetic hypothesis described in an unpublished paper and the synthesis of the generic delimitations within Theaceae, the proper name for each genus and their synonyms is provided. Key words: Theaceae, Taxonomy, genera.
\end{abstract}

a familia Theaceae D. Don está conformada por un grupo de plantas leñosas distribuidas en las regiones tropicales y subtropicales del mundo; en Europa sólo se han encontrado restos fósiles cuya determinación taxonómica se ha asignado a esta familia (Spongberg 1974; Grote y Dilcher 1989). Los caracteres por los cuales se les reconocía en la antigüedad sólo las caracterizaban de modo parcial y se incluían en ellas varios taxones alejados filogenéticamente, muchas veces por la única razón de presentar hojas alternas y muchos estambres. Keng (1962) afirmó que las Theaceae son una familia de difícil definición; este autor sostiene que uno de los únicos caracteres anatómicos que comparten los miembros de Theaceae es la presencia de esclereidas en la mayor parte de la planta. Metcalf y Chalk (1972) apoyan esta idea, sustentando que los idioblastos esclerenquimatosos en los tejidos parenquimatosos de la hoja, así como en la corteza y médula, son especialmente característicos de la familia. Schofield (1968) sustenta que los "Idioblasts are well-known features of the Theaceae, as are glands of the Guttiferae" (Los idioblastos son características bien conocidas de las Theaceae, como lo son las glándulas para las Guttiferae).

$\mathrm{El}$ interés por esta familia nace de una serie de estudios florístico-taxonómicos efectuados por la autora en diferentes áreas geográficas de México, en las cuales predominan los bosques mesófilos (Luna et al. 1988; Luna et al. 1989; Luna et al. 1994), donde sus miembros se encuentran frecuentemente. Otro punto 
de interés en las Theaceae radica en los patrones de distribución que exhiben algunos géneros, lo que destaca su relevancia biogeográfica, en especial la disyunción o vicarianza América-Asia (Rzedowski 1991; Weitzman 1995).

En general, se considera que entender las relaciones filogenéticas en los taxones constituyentes de un grupo mayor (Llorente y Espinosa 1992), como ocurre en Theaceae, permite generar hipótesis que contribuyan a la comprensión de los complejos patrones de distribución de esta familia, además de permitir una clasificación natural (Nelson y Platnick 1981).

Este trabajo sintetiza parte de los resultados de un análisis filogenético efectuado con los géneros de la familia (Luna y Weitzman en prep.) y tiene como objetivo presentar un resumen de los géneros de Theaceae reconocidos, indicando los caracteres por los cuales se les reconoce como grupos monofiléticos, así como bosquejar sus posibles relaciones intergenéricas. Cada género se evaluó críticamente con base en el examen de una serie de caracteres de tipo morfológico; en la mayoría de los casos se han podido determinar grupos naturales a nivel genérico; sin embargo, todavía algunos taxones requieren de estudios más profundos para delimitarlos con un mayor rigor metodológico como monofiléticos; tal es el caso de Ternstroemia y posiblemente de Eurya.

\section{Método}

La circunscripción genérica adoptada en este trabajo se basó en el examen exhaustivo de la literatura sobre Theaceae y grupos relacionados; se estudiaron varios centenares de ejemplares de herbario, para verificar los caracteres de todos los géneros, con excepción de Apterosperma Hung T. Chang y Euryodendron. Hung T. Chang, por carencia de material herborizado en los herbarios consultados. Para todos los géneros se examinaron la mayoría de las especies, particularmente tipos y siempre en un número mínimo de cuatro ejemplares por especie. Con base en el examen de la literatura, se eligieron los herbarios visitados y que cuentan con representación de la mayoría de las especies, éstos fueron: Herbario Nacional del Instituto de Biología, UNAM (MEXU), el herbario de los Jardines Botánicos Reales de Kew (K), el herbario del British Museum of Natural History, Londres (BM), el de la Universidad de Harvard, Cambridge, Massachussets (GH), el del Missouri Botanical Garden, Saint Louis, Missouri (MO), el del New York Botanical Garden, Bronx, New York (NY) y el Herbario Nacional de los Estados Unidos, en la Smithsonian. Institution, Washington, DC. (US).
Uno de los criterios utilizados conceptualmente para delimitar y definir una entidad taxonómica natural, sean géneros, familias u otros taxones, es aquel que considera a los caracteres como posibles sinapomorfias, los cuales definen al grupo como natural o monofilético. En este trabajo se analizaron los criterios utilizados hasta la fecha para la delimitación de los géneros en la familia, estudiando detalladamente las descripciones originales y los trabajos revisionales existentes, verificándolos en los ejemplares. En muchos casos se pudo consultar el material tipo de las especies de Theaceae, con lo cual se adquirió información de gran valor metodológico y de referencia para estudios taxonómicos. Además al estudiar material herborizado de importancia taxonómica fundamental se tiene la posibilidad de reconocer aquellos caracteres que realmente delimitan y definen a cada grupo genérico y las especies que lo constituyen.

Los géneros reconocidos en este trabajo reflejan la aplicación de los criterios antes mencionados y son congruentes con los resultados obtenidos en un análisis filogenético de la familia (Luna y Weitzman en prep.). Aunque aquí no se discuten tales resultados, se presentan para la mayoría de los géneros él o los caracteres exclusivos que definen su condición monofilética (autapomorfias) y que son la base para su reconocimiento como géneros en la familia.

\section{Resultados}

\section{Sintesis histórica}

La familia Theaceae fue concebida originalmente por Mirbel en 1813 (Keng 1962), aunque el Código Internacional de Nomenclatura Botánica (Greuter 1994) lista el nombre propuesto por D. Don (1825) como el nombre válido (nomen conservandum). Sin embargo, los primeros registros bibliográficos que se tienen se remontan a la época de Linneo (1753), quien reconoció cuatro taxones, ubicándolos en tres diferentes clases: Thea (T. sinensis en su clase Polyandria Monogynia), Camellia y Stewartia (C. japonica y S. malacodendron, ambas en su clase Monadelphia Polyandria) e Hypericum ( $H$. lasianthus en su clase Polyadelphia Polyandria).

Antes de que la familia fuera circunscrita (Don, 1825), varios autores ya habían descrito algunos de sus miembros, por ejemplo Adanson (1763: Mokof [ $=$ Ternstroemia], Tsubaki [=Camellia $]$ y Tsia $[=$ Camellia $]$ ), Mitchell (1769: Malachodendron [=Stewartia]), Ellis (1771: Gordonia), Aublet (1775: Taonabo [=Ternstroemia]), Scopoli (1777: Dupinia [=Ternstroemia] y Hoferia [=Ternstroemia $]$ ), Linneo hijo (1782: Visnea), Mutis (1782: Ternstroemia), Thunberg (1783: Cleyera, Eurya), 
Marshall (1785: Franklinia [=Gordonia]), Swartz (1788: Eroteum $[=$ Freziera] $)$, A. L. de Jussieu (1789: Mocanera [=Visnea $]$ ), Salisbury (1796: Cavanilla $[=$ Stewartia $]$ ) y Michauxia [=Gordonia]; 1805: Lacathea [=Gordonia]), Ruiz y Pavón (1794: Lettsomia [=Freziera]), Willdenow (1799: Freziera), Schrader (1821: Wikstroemia $[=$ Gordonia]) y Jack (1822: Adinandra).

A. P. de Candolle (1822) fue el primer autor en hacer una revisión de las Theaceae, a las que denominó Ternstroemiaceae. Este autor dividió a la familia en tres tribus: en la primera incluyó al género Ternstroemia, en la segunda a Cleyera, Freziera, Eurya y Lettsomia y en la tercera a Apatelia y Saurauja. Más tarde (de Candolle, 1824) agregó dos tribus a la familia, incluyendo, por un lado, a los géneros Cochlospermum, Laplacea y Ventenatia y por el otro, a Malachodendron, Stewartia y Gordonia; asimismo, segregó a Camellia y Thea en una familia diferente (Ordo Camellieae).

Kunth (1822) reunió como miembros de las Ternstroemiées a los géneros Cochlospermum, Ventenatia, Stewartia y Oncoba y como Theácées a Gordonia y Malachodendron.

Cambessèdes (1828) al elaborar una monografía de la familia, incluyó a 20 géneros dentro de las Ternstroemiacées: Cochlospermum, Ternstroemia, Cleyera, Freziera, Lettsomia, Eurya, Saurauia, Stewartia, Malachodendron, Laplacea, Gordonia, Camellia, Ventenatia, Bonnetia, Archytaea, Mahurea, Marila, Kielmeyera, Caraipa y Thea.

Muchos otros autores han estudiado a la familia; entre ellos destacan D. Don (1825), quien trabajó los taxones de Nepal y atribuyó en esa ocasión el nombre de la familia a de Candolle y A.L. Jussieu; Spach (1835), quien continuó con la separación de sus miembros en dos familias, Ternstroemiaceae y Camelliaceae; Endlicher (1843) quien incluyó 27 géneros en su tratamiento; Bentham y Hooker (1862), quienes tienen uno de los conceptos más amplios de la familia; Wawra (1866) quien trabajó los géneros de Brasil; Dyer (1872) quien lo hizo para la India y zonas adyacentes; Baillon (1873) quien consideró 28 taxones; Hemsley (1879-1888) quien hizo el tratamiento de los géneros para la Biologia Centrali-Americana; Szyszylowicz (1893) y Melchior (1925), quienes lo hicieron para las ediciones de la obra de Engler y Prantl, entre los más importantes.

La ubicación de Theaceae en diferentes órdenes ha oscurecido el entendimiento de sus relaciones con otras familias. Por ejemplo, para algunos ha sido miembro del orden Guttiferales (cuadro 1), otros la ubican en su propio orden Theales e incluso ha sido mencionada como parte del orden Parietales (Dalla Torre \& Harms 1963; Lawrence, 1951, quienes siguen el esquema propuesto por Engler 1908).
Algunas relaciones más estrechas de la familia Theaceae con otras familias han sido propuestas. Heywood (1979), por ejemplo, las relaciona con las Dipterocarpaceae, Guttiferae y Marcgraviaceae; Cronquist (1981), por su parte, las relaciona más estrechamente con las Dilleniaceae, en tanto que Goldberg (1986) las asocia principalmente con las Symplocaceae. Con base en la evidencia anatómica, Metcalf y Chalk (1972) y Schofield (1968) consideran que las Theaceae tienen una relación más estrecha con las Clusiaceae; Seetharam (1985) apoya esta relación con base en la evidencia palinológica.

A nivel genérico ha existido igualmente gran discusión acerca del número de miembros de la familia, con un flujo de géneros entre ella y otras familias como Actinidiaceae, Asteropeiaceae, Bonnetiaceae, Caryocaraceae, Clusiaceae, Cochlospermaceae, Marcgraviaceae, Ochnaceae, Pellicieraceae, Saurauiaceae y Tetrameristaceae.

Hasta el momento no ha habido un análisis filogenético que permita definir a las Theaceae con base en caracteres derivados compartidos. Un estudio, actualmente en desarrollo por Luna y Weitzman (en prep.), muestra que la familia está muy relacionada con las familias Clusiaceae y Bonnetiaceae sensu P. Stevens $(1996 a, b)$.

\section{Los géneros de Theaceae}

Como resultado del análisis bibliográfico y del estudio de ejemplares de herbario, se propone que la familia Theaceae está constituida por 20 géneros y por alrededor de 600 especies; cinco de los géneros aún pueden considerarse de posición incierta de acuerdo con un bosquejo filogenético realizado (Luna y Weitzman en prep.). En el cuadro 2 se presenta un resumen del número de géneros considerados por diferentes autores como parte de esta familia.

A continuación se listan los géneros reconocidos mencionando el número de especies que comprende cada uno, así como datos acerca de su distribución geográfica y relaciones sugeridas por otros autores. Se indica también en la mayoría de los casos, él o los caracteres que de acuerdo con el bosquejo filogenético alcanzado sugieren la condición monofilética de cada género. Los últimos cinco se listan provisionalmente como de posición incierta en las Theaceae.

\section{Adinandra Jack, Malayan Misc. 2 (7): 49. 1822.}

Sarosanthera Korth. in Temminck, Verh. nat. Gesch. Ned. Bezitt., Bot. 103. 1840.

Adinandra Jack sect. Symphiandra Szyszyl. in En- 
Cuadro 1. Órdenes en los que ha sido ubicada la familia Theaceae en diferentes sistemas de clasificación y familias que incluye cada uno

\begin{tabular}{|c|c|c|c|}
\hline Bentham \& Hooker (1862) & Bessey (1915) & Hutchinson (1973) & \\
\hline $\begin{array}{l}\text { Orden Guttiferales: } \\
\text { Chlaenaceae } \\
\text { Dipterocarpaceae } \\
\text { Elatinaceae } \\
\text { Guttiferae } \\
\text { Hypericaceae } \\
\text { Ternstroemiaceae } \\
\text { [=Theaceae] }\end{array}$ & $\begin{array}{l}\text { Orden Guttiferales: } \\
\text { Achariaceae } \\
\text { Bixaceae } \\
\text { Caricaceae } \\
\text { Caryocaraceae } \\
\text { Cistaceae } \\
\text { Cochlospermaceae } \\
\text { Dipterocarpaceae } \\
\text { Eucryphiaceae } \\
\text { Flacourtiaceae } \\
\text { Guttiferaceae } \\
\text { Koeberliniaceae } \\
\text { Malesherbiaceae } \\
\text { Marcgraviaceae } \\
\text { Passifloraceae } \\
\text { Quiinaceae } \\
\text { Stachyuraceae } \\
\text { Theaceae } \\
\text { Turneraceae } \\
\text { Violaceae }\end{array}$ & $\begin{array}{l}\text { Orden Theales: } \\
\text { Actinidiaceae } \\
\text { Bonnetiaceae } \\
\text { Caryocaraceae } \\
\text { Marcgraviaceae } \\
\text { Medusagynaceae } \\
\text { Pellicieraceae } \\
\text { Pentaphylacaceae } \\
\text { Saurauiaceae } \\
\text { Tetrameristaceae } \\
\text { Theaceae }\end{array}$ & \\
\hline Cronquist (1981) & Takhtajan (1980) & Dahlgren (1983) & Thorne (1992) \\
\hline $\begin{array}{l}\text { Orden Theales: } \\
\text { Actinidiaceae } \\
\text { Caryocaraceae } \\
\text { Clusiaceae } \\
\text { Dipterocarpaceae } \\
\text { Elatinaceae } \\
\text { Marcgraviaceae } \\
\text { Medusagynaceae } \\
\text { Ochnaceae } \\
\text { Oncothecaceae } \\
\text { Paracryphiaceae } \\
\text { Pellicieraceae } \\
\text { Pentaphylacaceae } \\
\text { Quiinaceae } \\
\text { Sarcolaenaceae } \\
\text { Scytopetalaceae } \\
\text { Sphaerosepalaceae } \\
\text { Tetrameristaceae } \\
\text { Theaceae }\end{array}$ & $\begin{array}{l}\text { Orden Theales: } \\
\text { Asteropeiaceae } \\
\text { Bonnetiaceae } \\
\text { Caryocaraceae } \\
\text { Clusiaceae } \\
\text { Marcgraviaceae } \\
\text { Oncothecaceae } \\
\text { Pellicieraceae } \\
\text { Pentaphylacaceae } \\
\text { Sladeniaceae } \\
\text { Stachyuraceae } \\
\text { Symplocaceae } \\
\text { Tetrameristaceae } \\
\text { Theaceae }\end{array}$ & $\begin{array}{l}\text { Orden Theales: } \\
\text { Ancistrocladaceae } \\
\text { Bonnetiaceae } \\
\text { Caryocaraceae } \\
\text { Clusiaceae } \\
\text { Dioncophyllaceae } \\
\text { Elatinaceae } \\
\text { Lecythidaceae } \\
\text { Marcgraviaceae } \\
\text { Medusagynaceae } \\
\text { Nepenthaceae } \\
\text { Ochnaceae } \\
\text { Oncothecaceae } \\
\text { Pentaphylacaceae } \\
\text { Quiinaceae } \\
\text { Scytopetalaceae } \\
\text { Stachyuraceae } \\
\text { Strasburgeriaceae } \\
\text { Theaceae }\end{array}$ & $\begin{array}{l}\text { Orden Theales: } \\
\text { Actinidiaceae } \\
\text { Ancistrocladaceae } \\
\text { Aquifoliaceae } \\
\text { Asteropeiaceae } \\
\text { Bonnetiaceae } \\
\text { Caryocaraceae } \\
\text { Chrysobalanaceae } \\
\text { Clethraceae } \\
\text { Clusiaceae } \\
\text { Cyrillaceae } \\
\text { Dilleniaceae } \\
\text { Dioncophyllaceae } \\
\text { Elatinaceae } \\
\text { Lecythidaceae } \\
\text { Marcgraviaceae } \\
\text { Medusaginaceae } \\
\text { Nepenthaceae } \\
\text { Ochnaceae } \\
\text { Oncothecaceae } \\
\text { Paracryphiaceae } \\
\text { Pellicieraceae } \\
\text { Pentaphylacaceae } \\
\text { Phellinaceae } \\
\text { Quiinaceae } \\
\text { Sarraceniaceae } \\
\text { Scytopetalaceae } \\
\text { Sphenostemonaceae } \\
\text { Stachyuraceae } \\
\text { Strasburgeriaceae } \\
\text { Symplocaceae } \\
\text { Tetrameristaceae } \\
\text { Theaceae }\end{array}$ \\
\hline
\end{tabular}


gler \& Prantl, Nat. Pflanzenfam. 3 (6): 189. 1893. Tsou (1995) sugiere que este taxón se diferencia de grupos relacionados por su placenta expandida, condición que posiblemente pruebe la monofilia del género.

Szyszylowicz (1893) separó al género Adinandra en tres secciones, dependiendo de la disposición de los estambres: Eleutherandra (con estambres libres, actualmente reconocida como el género Balthasaria), Adinandra (con estambres en haces) y Symphiandra (con estambres unidos y dispuestos en un tubo). Melchior (1925) agregó una cuarta sección, Eleutherostyla, reconocida actualmente como un género aparte (Archboldiodendron), con base en los estilos libres.

Relaciones: este género se ha relacionado con Archboldiodendron y Balthasaria.

Diversidad: aproximadamente 76 especies.

Distribución: sureste de Asia, incluyendo el Archipiélago Malayo; Sri Lanka; Nueva Guinea (Papuasia).

Referencia: Kobuski (1947).

2. Anneslea Wall., Pl. asiat. rar. 1: 5, t. 5. 1829, nom. cons., non Anneslia Salisb., 1807 (Fabaceae).

Annesleia Spach in Orbigny, Dict. univ. hist. nat. 1: 546. 1841, orth. mut.

Richtera Rchb., Deut. Bot. Herb.-Buch 208. 1841, nom. nud.

Callosmia C. Presl, Abh. k. böhm. Ges. Wiss. ser. 5, 3: 533. 1845.

Daydonia Britten, J. Bot. 26: 11. 1888.

Mountnorrisia Szyszyl. in Engler \& Prantl, Nat. Pflanzenfam. 3(6): 189. 1893.

Annesleya T. Post \& O. Kuntze, Nomenkl. Revis. höher. Pfl.-Gr. 32. 1900, orth. mut.

Paranneslea Gagnep., Bull. Soc. Bot. France 95: 29. 1948.

La monofilia del grupo puede postularse con base en los pétalos en forma de reloj de arena, esto es, profundamente constreñidos por la mitad.

Kobuski (1952a) cita seis sinónimos para este género, cinco de los cuales pertenecen claramente a él. Uno de ellos, Richtera, fue listado por Reichenbach (1841 en: Kobuski 1952a) sin ninguna descripción o referencia a material de herbario. Aún recientemente es posible encontrar ejemplares de herbario bajo el epíteto de Paranneslea, género descrito por Gagnepain en 1948, que para Kobuski (1952a) corresponde bien con Anneslea fragrans Wall.

Relaciones: este género se ha relacionado con Ternstroemia.

Diversidad: 2 o 3 especies.

Distribución: Asia tropical y subtropical, desde el sur de China, incluyendo a Hainan y Taiwan, hasta Su- matra en el Archipiélago Malayo.

Referencia: Kobuski (1952a).

3. Archboldiodendron Kobuski, J. Arnold Arbor. 21: 140. 1940.

Adinandra Jack sect. Eleutherostyla Melch. in Engler \& Prantl, Nat. Pflanzenfam. ed. 2. 21: 145. 1925.

Este género se propone como grupo monofilético con base en la existencia de los pétalos en dos series y los tricomas naranja-amarillos que cubren abundamentemente las partes vegetativas.

Este taxón fue segregado de Adinandra, en particular de una de las secciones propuestas por Szyszylowicz (1893) para el género (sección Eleutherostyla), por su característica de presentar los estilos libres.

Relaciones: género segregado de Adinandra, con el que posiblemente esté más estrechamente relacionado. Diversidad: género monotípico (A. calocericeum Kobuski).

Distribución: Nueva Guinea (Papuasia).

Referencias: Kobuski (1940); Barker (1980).

4. Balthasaria Verdc., Kew Bull. 23: 469. 1969.

Adinandra Jack sect. Eleutherandra Szyzyl. in Engler \& Prantl, Nat. Pflanzenfam. 3 (6): 189. 1893. Adinandropsis Pitt-Schenkel, J. Ecol. 26: 80. 1938, nom. nud.

Melchiora Kobuski, J. Arnold Arbor. 37: 154. 1956, non Melchioria Penzig \& Saccardo, 1969 (Fungi: Sphaeriales).

Se postula como grupo monofilético con base en los lóbulos de la corola naranjas o rojos, en una relación 3:1 con respecto a los lóbulos del cáliz.

Las especies de este género fueron descritas como parte de Adinandra, del que fueron después segregadas y nombradas bajo Melchiora. El nombre Melchiora Kobuski (1956) fue sustituido por Balthasaria (Verdcourt 1969), para evitar confusión con el género Melchioria Penzig \& Saccardo, que corresponde a un grupo de hongos (Sphaeriales).

Relaciones: género relacionado con Adinandra.

Diversidad: 3 especies.

Distribución: Africa (Tanganika, Congo Belga, Uganda). Referencias: Kobuski (1956, 1957); Verdcourt (1969).

5. Camellia L., Sp. pl. 698. 1753.

Thea L., Sp. pl. 515. 1753.

Tsubaki Adans., Fam. pl. 2: 399. 1763.

Tsia Adans., Fam. pl. 2: 450. 1763.

Calpandria Blume, Bijdr. 178. 1825. 
Theaphylla Raf., Med. fl. 2: 267. 1830.

Sasanqua Nees in Siebold, Nippon 4: 13. 1833-4.

Theaphyla Raf., Fl. tellur. 1: 17. 1837, orth. mut.

Kemelia Raf., Sylva tellur. 139. 1838.

Demitus Raf., Sylva tellur. 139. 1838.

Drupifera Raf., Sylva tellur. 140. 1838.

Piquetia Hallier f., Beih. Bot. Centralbl. 39(2): 162. 1921.

Stereocarpus Hallier f., Beih. Bot. Centralbl. 39(2): 162. 1921.

Camelliastrum Nakai, J. Jap. Bot. 16: 699. 1940.

Theopsis Nakai, J. Jap. Bot. 16: 704. 1940.

Yunnanea Hu, Acta Phytotax. Sin. 5: 282. 1956.

Kailosocarpus Hu, Scientia (China) 170. 1957, nom.

nud.

Paräpiquetia $\mathrm{Hu}$, Scientia (China) 170. 1957, nom. nud.

Glyptocarpa Hu, Acta Phytotax. Sin. 10: 25. 1965. Las características que definen la monofilia de este género son el endospermo con alto contenido de aceite y posiblemente la formación del saco embrionario tipo Allium.

Se considera que antes de 1930 sólo había un conocimiento elemental de este género. Más recientemente, Chang (1981) en su monografía de Camellia considera cuatro subgéneros: 1) Protocamellia, con tres secciones; 2) Camellia, con siete secciones; 3) Thea con ocho secciones y 4) y Metacamellia con dos secciones. La obra de este autor fue traducida del chino al inglés por Bartholomew en 1984 (Chang y Bartholomew, 1984). Este es el género más diversificado de Theaceae y de acuerdo con Chang (1981) es también el más primitivo, ya que en él están representados los caracteres más plesiomórficos de la familia. Para este género existen un número importante de sinónimos, muchos de los cuales corresponden actualmente a las secciones de Chang (1981).

Relaciones: género relacionado con Pyrenaria.

Diversidad: alrededor de 200 especies.

Distribución: este de la India y sur de China, Indomalasia, Japón y Filipinas.

Referencias: Chang (1981); Chang y Bartholomew (1984).

6. Cleyera Thunb., Nov. gen. pl. 3: 68. 1783, nom. cons., non Cleyera Adans., 1763 (Loganiaceae).

Tristylium Turcz., Bull. Soc. Imp. Naturalistes Moscou 31 (1): 247. 1858.

Eurya sect. Cleyera Szyszyl. in Engler \& Prantl, Nat. Pflanzenfam. 3(6): 189. 1893.

Eurya subg. Cleyera Melch. in Engler \& Prantl, Nat. Pflanzenfam. ed. 2. 21: 147. 1925.

Sakakia Nakai, Fl. sylv. kor. 17: 76. 1928.
Las anteras setosas al parecer son un rasgo característico de este género, mismas que pueden considerarse como la apomorfia que define su condición monofilética.

El material original en que Thunberg (1783) basó su descripción de Cleyera contiene elementos de dos plantas diferentes. Thunberg no se dio cuenta de ello sino hasta nueve años después. Cuando se percató de su error, confirmó que la mayor parte del ejemplar correspondía a Cleyera japonica, la especie tipo; la otra porción del ejemplar la ubicó dentro del género Ternstroemia (Ternstroemia gymnanthera (W. \& A.) Sprague) (Kobuski 1937). Más tarde Szyszylowicz (1893) consideró a este taxón como una sección de Eurya y Melchior (1925) como un subgénero también de Eurya. Desde entonces los botánicos han tenido problemas al utilizar los nombres de Cleyera y Eurya; no obstante, según Kobuski (1937) son lo suficientemente diferentes para ser reconocidos como entidades distintas. Por lo anterior, algunos representantes de Cleyera de América han sido incluidos dentro del género Eurya (Szyszylowicz 1893; Melchior 1925), dentro de Freziera (Tulasne 1847; Bentham 1861) o en Cleyera (Choisy 1855; Kobuski 1941b, entre muchos otros). Urban (1896) sugirió que Cleyera y Freziera debían retenerse como géneros diferentes. Algunas clasificaciones antiguas (por ejemplo, Szyszylowicz 1893; Melchior 1925) no incluyen ni a Freziera ni a Cleyera, géneros aceptados en la actualidad, sino que los consideran como subgéneros de Eurya.

Relaciones: género relacionado con Eurya y Freziera. Diversidad: alrededor de 17 especies; 7 en el neotrópico (Weitzman 1995).

Distribución: trópicos y subtrópicos del mundo: este de Asia (Japón, Corea, Taiwan, China e India) y América (oeste y sur de México a Centroamérica, incluyendo a las Grandes Antillas).

Referencias: Kobuski (1937, 1941b); Weitzman (1987).

7. Eurya Thunb., Nov. gen. pl. 3: 67. 1783.

Geeria Blume, Bijdr. 124. 1825.

Ternstroemiopsis Urb., Ber. Deutsch. Bot. Ges. 14: 49. 1896.

Pseudoeurya Yamam., J. Soc. Trop. Agric. 5: 351. 1933.

Tsou (1995) sugiere que las especies de este género presentan una sola capa media en la pared de la antera, tal vez como resultado del decremento en el tamaño de la flor; posiblemente esta característica pueda postularse como la apomorfia que defina su condición monofilética.

Este género ha tenido serios problemas en la delimitación de sus especies y en su sinonimia. El princi- 
pal problema es que dentro de este taxón fueron incluidos Cleyera y Freziera, entidades que actualmente se reconocen como géneros distintos. Por ejemplo, Szyszylowicz (1893) incluyó dentro de este género a tres secciones: Cleyera, Freziera y Proteurya; Urban (1896) estableció un año después un nuevo género, Ternstroemiopsis y consideró a Freziera, Cleyera y Eurya como géneros separados. Kobuski (1935) continuó con la tendencia de Urban de considerar a Freziera y Cleyera como entidades independientes de Eurya; este trabajo coincide con el criterio de Kobuski (1935).

Relaciones: se le ha relacionado con Cleyera y Freziera. Diversidad: de 80 a 140 especies.

Distribución: este de Asia, desde el sur de China a través de Indomalasia hasta Indonesia; Nueva Guinea (Papuasia) e Islas Hawaii.

Referencias: Kobuski (1935, 1938); Ling (1966).

8. Freziera Willd., Sp. pl. 2 (2): 1179. 1799, nom. cons.

Lettsomia Ruiz \& Pav., Fl. peruv. prodr. 4: 77. 1784, non Letsomia Roxb. 1814 (Convolvulaceae).

Eroteum Sw., Prodr. 5: 85. 1788.

Fresiera Mirb., Nouv. Bull. Sci. Soc. Philom. Paris 1813: 381. 1813, orth. mut.

Patascoya Urb., Ber. Deutsch. Bot. Ges. 14: 283. 1896.

Eurya subg. Freziera Melch. in Engler \& Prantl, Nat. Pflanzenfam. ed. 2. 21: 148. 1925.

Killipiodendron Kobuski, J. Arnold Arbor. 23: 231. 1942.

Weitzman (1987) propuso que la monofilia del género puede sustentarse en la corola urceolada y los sépalos sin seta terminal; no obstante, estas características no se presentan en algunas especies.

Género considerado en algún tiempo como parte de Eurya; sin embargo Urban (1896) lo ubica como una entidad distinta. Weitzman (1987) considera por su parte a Patascoya Urb. y Killipiodendron Kobuski como sinónimos de Freziera, criterio que es seguido en este trabajo.

Relaciones: género relacionado con Eurya.

Diversidad: 58 especies (Weitzman 1995).

Distribución: del sur de México al norte de Sudamérica, hasta el centro de Perú, incluyendo a las Antillas. Referencias: Kobuski (1941c); Weitzman (1987).

9. Gordonia J. Ellis, Philos. Trans. 60: 520, t. 11. 1771, nom. cons.

Lasianthus Adans., Fam. pl. 2: 398. 1763, non Lasianthus Jack, 1823 (Rubiaceae).

Franklinia Marshall, Arbust. amer. 48. 1785.

Gordona Cothen., Disp. veg. meth. 26. 1790, orth. mut.
Franklina J.F. Gmel., Syst. nat. 2: 775. 1791, orth. mut.

Michauxia Salisb., Prodr. stirp. Chap. Allerton 386. 1796, non Michauxia L'Hér., 1788 (Campanulaceae).

Lacathea Salisb., Parad. lond. 56, t. 56. 1805.

Wikstroemia Schrad., Hort. gott. 71. 1811.

Lindley $a$ Nees, Flora 4: 299. 1821, non Lindley $a$ Kunth, 1824 (Rosaceae).

Laplacea Kunth, in H.B.K., Nov. gen. sp. 5: 161. 1822 [folio], 5: 207. 1822 [quarto].

Haemocharis Salisb. ex Mart. \& Zucc., Nov. gen. sp. 1: 106. 1826 [1824].

Polyspora Sweet ex G. Don, Gen. Hist. 1: 564, 574. 1831.

Antheeischima Korth. in Temminck, Verh. nat. Gesch. Ned. Bezitt., Bot. 137. 1842.

Closaschima Korth. in Temminck, Verh. nat. Gesch. Ned. Bezitt., Bot. 137. 1842.

Carria Gardner, Calcutta J. Nat. Hist. 7: 6. 1846. Antheishma Hassk., Flora 30: 661. 1847, orth. mut. Dipterosperma Griff., Not. pl. asiat. 4: 564. 1854, non Dipterosperma Hassk., 1842 (Bignoniaceae).

Antheischima Benth. in Bentham \& Hooker, Gen. pl. 1: 186. 1862, orth. mut.

Nabiasodendron. Pit., Actes Soc. Linn. Bordeaux 57: 54. 1902.

Se propone como autapomorfia del género la cápsula oblongo-elipsoide que abre del ápice hacia la base.

Este taxón incluye en su sinonimia algunos nombres que todavía se utilizan, por ejemplo Laplacea, género que en ocasiones ha sido mantenido como una entidad distinta de Gordonia (cf. Kobuski 1949, 1950, 1951a; Brummitt 1992, entre otros) y Franklinia.

Relaciones: se le ha relacionado con Schima.

Diversidad: aproximadamente 70 especies; tres en el neotrópico (Weitzman 1995).

Distribución: trópicos y subtrópicos del mundo: en el sureste de Asia, desde la India y sur de China, Archipiélago Malayo, hasta Nueva Guinea; en América en el sureste de Estados Unidos y del sur de México al norte de Sudamérica, incluyendo a las Antillas.

Referencias: Burkill (1917); Kobuski (1951a); Keng (1980a, 1984).

\section{Pyrenaria Blume, Bijdr. 1119. 1825.}

Eusynaxis Griff., Not. pl. asiat. 4: 560, t. 603. 1854. Tutcheria Dunn, J. Bot. 46: 324. 1908.

Sinopyrenaria Hu, Acta Phytotax. Sin. 5: 281. 1956.

Parapyrenaria Hung T. Chang, Acta Phytotax. Sin. 8: 287. 1963.

La monofilia de este.género puede ser postulada con base en el tipo de semilla y características del embrión, 
que según Keng (1972) son únicos dentro la familia Theaceae.

Blume (1825) al describir el género destacó su parecido con las Rosaceae, principalmente por el fruto semejante a un pomo con cinco lóculos, cada uno conteniendo dos "pirenos" con una semilla (de allí el nombre de Pyrenaria). Este fruto sólo superficialmente parece una pera o manzana, pero se desarrolla a partir de un ovario súpero; los supuestos "pirenos" descritos por Blume son semillas verdaderas (Keng 1980b).

Relaciones: género relacionado con Camellia.

Diversidad: 30 especies.

Distribución: sureste de Asia, desde el este de la India y sur de China, Taiwan e islas Riukiu, Archipiélago Malayo, hasta Indonesia (Borneo, Sumatra y Java). Referencia: Keng (1972, 1980b).

11. Schima Reinw. ex Blume, Cat. Buitenzorg 80. 1823.

La monofilia del género se propone con base en su característica cápsula globosa, con semillas reniformes aladas a todo alrededor, pero principalmente en la parte posterior.

La única especie de este género es, según Bloembergen (1952), una especie polimórfica, la cual incluye nueve subespecies y tres variedades.

Relaciones: género relacionado con Gordonia.

Diversidad: género monotípico ( $S$. wallichii (DC.) Korth.).

Distribución: este de Asia, principalmente en el Archipiélago Indomalayo.

Referencias: Airy-Shaw (1936); Bloembergen (1952).

12. Stewartia L., Sp. pl. 698. 1753.

Malachodendron J. Mitch., Diss. brev. bot. zool. 38. 1769.

Malachodendrum Juss., Gen. pl. 275. 1789, orth. mut.

Stuartia L'Hér., Stirp. nov. 153. 1791, orth. mut.

Stewarta Cothen., Disp. veg. meth. 26. 1790, orth. mut.

Cavanilla Salisb., Prodr. stirp. Chap. Allerton 385. 1796, non Cavanilla J.F. Gmel., 1791 (Sterculiaceae).

Hartia Dunn, Hooker's Icon. Pl. 2727. 1902.

Se propone como autapomorfia del género la presencia de esclereidas solamente en el pecíolo.

Este género tiene varios sinónimos: Malachodendron, mal atribuido a Cavanilles (1788 en: Spongberg 1974), fue descrito por Mitchell en 1769 (en Spongberg 1974); algunos autores durante el siglo XIX aceptaron a Malachodendron como un género diferente a Stewartia, $v$ gr. de Candolle (1824), quien reconoció a ambos en su estudio sobre las Ternstroemiaceae, al igual que Choisy (1855). Este último autor los incorporó en la tribu Stewartieae. Más tarde Dunn (1902 en: Spongberg 1974) propuso un género nuevo, Hartia, con base en en gran cantidad de caracteres, que más tarde fueron considerados como variación intraespecífica (Keng 1962; Spongberg 1974).

Relaciones: género relacionado con Camellia, Pyrenaria, Schima y Gordonia.

Diversidad: alrededor de 8 especies.

Distribución: este y sureste de Asia y este de Estados Unidos.

Referencias: Spongberg (1974); Yan (1981); Ye (1982); Kobuski (1951a).

13. Symplococarpon Airy Shaw, Hooker's Icon. Pl. 34: 3342. 1939.

La condición monofilética del género se postula con base en el fruto indehiscente, subgloboso, semejante en forma al del género Symplocos (Symplocaceae), bilocular y con dos semillas.

Este género ha sido confundido con Cleyera, sólo diferenciándose por la posición del ovario (súpero en Cleyera e ínfero en Symplococarpon).

Relaciones: género relacionado con Cleyera y con Freziera.

Diversidad: género monotípico (S. hintonii Airy Shaw). Distribución: del sur de México al norte de Sudamérica.

Referencias: Airy Shaw (1939); Kobuski (1941a).

14. Ternstroemia Mutis ex L.f, Suppl. pl. 39: 264. 1782, nom. cons.

Taonabo Aubl., Hist. pl. Guiane 569. 1775.

Dupinia Scop., Intr. hist. nat. 195. 1777.

Hoferia Scop., Intr. hist. nat. 194. 1777.

Tonabea Juss., Gen. pl. 262. 1789, orth. mut.

Reinwardtia Korth. in Temminck, Verh. nat. Gesch. Ned. Bezitt., Bot. 101. 1840.

Llanosia Blanco, Fl. Filip. ed. 2. 319. 1845.

Erythrochiton Griff., Proc. Linn. Soc. London 1: 282.

1846, non Erythrochiton Nees \& Mart., 1823 (Rutaceae).

Voelckeria Klotzsch \& H. Karst. ex Endl., Gen. suppl. 5: 66. 1850.

Mokofua O. Kuntze, Revis. gen. pl. 1: 63. 1891.

Adinandrella Exell, J. Bot. 65 (Suppl. 1): 30. 1927.

Por el momento no es posible determinar una característica que defina la condición monofilética de este taxón, dado que es un género sumamente heterogéneo y necesita ser reevaluado.

Este género ha tenido un gran número de sinónimos 
como Taonabo Aublet (1775), que fue previo a Ternstroemia; todavía en algunos herbarios es posible encontrar ejemplares bajo este nombre. El epíteto Ternstroemia fue adoptado por el Código Internacional de Nomenclatura como nomen conservandum. Kobuski (1942) separó a las especies neotropicales en tres grupos geográficos: de México a Centroamérica, Antillas y Sudamérica, todas ellas hermafroditas. Más tarde, al trabajar las especies filipinas (Kobuski 1961b) observó que las especies de esta región pueden ser dioicas, androdioicas o tal vez hermafroditas. Ese mismo año, también trabajó las especies de África (Kobuski 1961a) cambiando radicalmente su descripción del género.

Relaciones: género relacionado con Anneslea.

Diversidad: 110-160 especies; 50-70 especies en el neotrópico (Weitzman 1995).

Distribución: trópicos y subtrópicos del mundo: en el sureste de Asia, desde el sur de China, Taiwan, Sri Lanka, Japón, Archipiélago Malayo hasta Indonesia; Nueva Guinea (Papuasia); noroeste de Australia; en América desde México al norte de Sudamérica, hasta el sur del Brasil y centro de Bolivia, incluyendo a las Antillas; en África de Tanganika a Angola.

Referencias: Kobuski (1942, 1943, 1961a, 1961b)

\section{Visnea L.f., Suppl. pl. 36: 251. 1782.}

Mocanera A.L. Juss., Gen. pl. 318. 1789, non $M o-$ canera Blanco, 1837.

Se postula como posible apomorfia del género la posición del ovario, no homóloga a la de Symplococarpon y Anneslea.

La posición del género Visnea ha sido muy discutida; Endlicher (1836-1840) primero lo colocó dentro de las Theaceae, pero más tarde en el segundo suplemento de la misma publicación cambió de opinión refiriéndolo a las Ebenaceae. Johnson (1857 en: Kobuski 1952b) sugirió que existe relación entre este género y Clethra L. y opinó que Visnea podría ser una conexión entre las Ericaceae y Vaccinaceae; este mismo autor más tarde (1895 en: Kobuski 1952b) cambió de opinión, colocando al género dentro de las Theaceae.

Para finales del siglo XIX la mayoría de los botánicos aceptaron que Visnea pertenecía a las Theaceae. Szyszylowicz (1893) y Melchior (1925) lo colocaron en la tribu Ternstroemieae junto con Adinandra, Ternstroemia y Eurya.

Relaciones: se le ha relacionado con los miembros de la subfamilia Ternstroemioideae de Keng (1962).

Diversidad: género monotípico ( $V$. mocanera L.).

Distribución: Islas Canarias y Madeira.

Referencia: Kobuski (1952b).

\section{Géneros de posición incierta dentro de Theaceae}

16. Apterosperma Hung T. Chang, Acta Sci. Nat. Univ. Sunyatseni 1976 (2): 91. 1976.

Género poco conocido, sin ejemplares depositados en alguno de los herbarios consultados. Según el análisis filogenético realizado (Luna y Weitzman en prep.), es un taxón relacionado principalmente con Camellia, donde tal vez pueda incluirse. Por esta razón no se postula apomorfia para el género.

Relaciones: Chang (1976) considera que es un género cercano a Schima.

Diversidad: Género monotípico (A. ovata Hung T. Chang).

Distribución: sur de China.

Referencia: Chang (1976).

17. Dankia Gagnep. in Humbert, Suppl. Fl. gén. Indochine 1: 198. 1939.

Con base en la falta de material herborizado no es posible determinar la apomorfia del género. Según el análisis filogenético realizado (Luna y Weitzman en prep.) es un taxón relacionado con Camellia, Schima y Pyrenaria. Género monotípico, descrito por Gagnepain (1939) como perteneciente a las Flacourtiaceae. Por algunas cartas encontradas en los herbarios de R. Sealy, C. E. Kobuski y H. Sleumer, se sabe que dichos autores opinan que este taxón corresponde bien con el tipo de Camellia depositado en P. Phamhoang (1991) sigue este criterio, citándolo para la Flora de Vietnam como Camellia langbianensis (Gagnep.) Phamhoang. Desafortunadamente no hay material herborizado en los herbarios consultados; sólo se encontró un ejemplar estéril en $\mathrm{K}$.

Relaciones: género relacionado con (o posiblemente parte de) Camellia.

Diversidad: género monotípico (D. langbianensis Gagnep.).

Distribución: Indochina.

Referencias: Gagnepain (1939); Phamhoang (1991).

18. Euryodendron Hung T. Chang, Acta Sci. Nat. Univ. Sunyatseni 1963 (4): 129. 1963.

Lishichenia Hung T. Chang, Sunyatsenia 1: 6, 23, 31. 1962, nom. nud.

Género poco conocido, sin material depositado en los herbarios consultados; sólo existe un fragmento en US. Con base en el análisis filogenético efectuado (Luna y Weitzman en prep.) se confirma su relación con Eurya y Cleyera, aunque aún no ha sido posible reconocer la apomorfia que determine la condición 
Cuadro 2. Géneros reconocidos en la familias Theaceae por diversos autores

\begin{tabular}{|c|c|c|c|}
\hline De Candolle (1824) & Don (1831) & Spach (1835) & Endlicher (1843) \\
\hline $\begin{array}{l}\text { Apatelia } \\
\text { Camellia } \\
\text { Cleyera } \\
\text { Cochlospermum } \\
\text { Eurya } \\
\text { Freziera } \\
\text { Gordonia } \\
\text { Laplacea } \\
\text { Lettsomia } \\
\text { Malachodendron } \\
\text { Saurauia } \\
\text { Stewartia } \\
\text { Ternstroemia } \\
\text { Thea } \\
\text { Ventenatia }\end{array}$ & $\begin{array}{l}\text { Anneslea } \\
\text { Apatelia } \\
\text { Architaea } \\
\text { Bonnetia } \\
\text { Blumea } \\
\text { Camellia } \\
\text { Caraipa } \\
\text { Cleyera } \\
\text { Cochlospermum } \\
\text { Eurya } \\
\text { Freziera } \\
\text { Geeria } \\
\text { Godoya } \\
\text { Gordonia } \\
\text { Kielmiera } \\
\text { Laplacea } \\
\text { Lettsomia } \\
\text { Mahurea } \\
\text { Malachodendron } \\
\text { Marila } \\
\text { Polyspora } \\
\text { Saurauja } \\
\text { Schima } \\
\text { Stuartia } \\
\text { Ternstroemia } \\
\text { Thea } \\
\text { Ventenatia }\end{array}$ & $\begin{array}{l}\text { Architaea } \\
\text { Bonnetia } \\
\text { Camellia } \\
\text { Caraipa } \\
\text { Cleyera } \\
\text { Cochlospermum } \\
\text { Eurya } \\
\text { Freziera } \\
\text { Geeria } \\
\text { Gordonia } \\
\text { Kielmeyera } \\
\text { Laplacea } \\
\text { Lettsomia } \\
\text { Mahurea } \\
\text { Malachodendron } \\
\text { Reinwardtia } \\
\text { Saurauia } \\
\text { Stewartia } \\
\text { Ternstroemia } \\
\text { Thea } \\
\text { Ventenatia } \\
\text { Wickstroemia }\end{array}$ & $\begin{array}{l}\text { Adinandra } \\
\text { Anneslea } \\
\text { Archytaea } \\
\text { Bonnetia } \\
\text { Camellia } \\
\text { Caraipa } \\
\text { Cleyera } \\
\text { Cochlospermum } \\
\text { Dicalyx } \\
\text { Eurya } \\
\text { Euryanthe } \\
\text { Freziera } \\
\text { Godoya } \\
\text { Gordonia } \\
\text { Kielmeyera } \\
\text { Laplacea } \\
\text { Lettsomia } \\
\text { Leucoxylon } \\
\text { Marila } \\
\text { Mahurea } \\
\text { Microsemma } \\
\text { Pyrenaria } \\
\text { Saurauja } \\
\text { Stuartia } \\
\text { Ternströmia } \\
\text { Thea } \\
\text { Visnea }\end{array}$ \\
\hline Bentham \& Hooker (1862) & Baillon (1873) & Szyszylowicz (1893) & \\
\hline $\begin{array}{l}\text { Actinidia } \\
\text { Adinandra } \\
\text { Anneslea } \\
\text { Anthodiscus } \\
\text { Archytaea } \\
\text { Bonnetia } \\
\text { Camellia } \\
\text { Caraipa } \\
\text { Caryocar } \\
\text { Cleyera } \\
\text { Eurya } \\
\text { Freziera } \\
\text { Gordonia } \\
\text { Haploclathra } \\
\text { Kielmeyera } \\
\text { Laplacea } \\
\text { Mahurea } \\
\text { Marcgravia } \\
\text { Marila } \\
\text { Microsemma } \\
\text { Norantea } \\
\text { Omphalocarpum }\end{array}$ & $\begin{array}{l}\text { Adinandra } \\
\text { Anneslea } \\
\text { Anthodiscus } \\
\text { Archytaea } \\
\text { Bonnetia } \\
\text { Caraipa } \\
\text { Caryocar } \\
\text { Eroteum } \\
\text { Eurya } \\
\text { Gordonia } \\
\text { Haemocharis } \\
\text { Haploclathra } \\
\text { Kielmeyera } \\
\text { Mahurea } \\
\text { Marcgravia } \\
\text { Marila } \\
\text { Microsemma } \\
\text { Norantea } \\
\text { Pelliciera } \\
\text { Poeciloneuron } \\
\text { Pyrenaria } \\
\text { Ruyschia }\end{array}$ & $\begin{array}{l}\text { Adinandra } \\
\text { Archytaea } \\
\text { Asteropeia } \\
\text { Bonnetia } \\
\text { Eurya } \\
\text { Gordonia } \\
\text { Haemocharis } \\
\text { Mountnorrisia } \\
\text { Pelliciera } \\
\text { Pyrenaria } \\
\text { Schima } \\
\text { Stewartia } \\
\text { Taonabo } \\
\text { Thea } \\
\text { Tremanthera } \\
\text { Visnea }\end{array}$ & \\
\hline
\end{tabular}




\begin{tabular}{|c|c|c|c|}
\hline Bentham \& Hooker (1862) & Baillon (1873) & Szyszylowicz (1893) & \\
\hline $\begin{array}{l}\text { Pelliciera } \\
\text { Pentaphylax } \\
\text { Pyrenaria } \\
\text { Ruyschia } \\
\text { Saurauja } \\
\text { Schima } \\
\text { Stachyurus } \\
\text { Stuartia } \\
\text { Ternstroemia } \\
\text { Visnea }\end{array}$ & $\begin{array}{l}\text { Saurauja } \\
\text { Schima } \\
\text { Steuartia } \\
\text { Thea } \\
\text { Ternstroemia } \\
\text { Visnea }\end{array}$ & & \\
\hline Melchior (1924) & Keng (1962) & Brummitt (1992) & Este trabajo \\
\hline $\begin{array}{l}\text { Adinandra } \\
\text { Anneslea } \\
\text { Archytaea } \\
\text { Asteropeia } \\
\text { Bonnetia } \\
\text { Camellia } \\
\text { Eurya } \\
\text { Franklinia } \\
\text { Gordonia } \\
\text { Hartia } \\
\text { Laplacea } \\
\text { Patascoya } \\
\text { Pelliciera } \\
\text { Piquetia } \\
\text { Ploiarum } \\
\text { Pyrenaria } \\
\text { Schima } \\
\text { Stereocarpus } \\
\text { Stewartia } \\
\text { Ternstroemia } \\
\text { Tetramerista } \\
\text { Tutcheria } \\
\text { Visnea }\end{array}$ & $\begin{array}{l}\text { Adinandra } \\
\text { Anneslea } \\
\text { Archboldiodendron } \\
\text { Camellia } \\
\text { Cleyera } \\
\text { Eurya } \\
\text { Franklinia } \\
\text { Gordonia } \\
\text { Hartia } \\
\text { Killipiodendron } \\
\text { Laplacea } \\
\text { Melchiora } \\
\text { Patascoya } \\
\text { Piquetia } \\
\text { Pyrenaria } \\
\text { Schima } \\
\text { Sladenia } \\
\text { Stuartia } \\
\text { Symplococarpon } \\
\text { Stereocarpus } \\
\text { Ternstroemia } \\
\text { Tutcheria } \\
\text { Visnea } \\
\text { Yunnanea }\end{array}$ & $\begin{array}{l}\text { Adinandra } \\
\text { Anneslea } \\
\text { Apterosperma } \\
\text { Archboldiodendron } \\
\text { Balthasaria } \\
\text { Camellia } \\
\text { Cleyera } \\
\text { Dankia } \\
\text { Eurya } \\
\text { Ficalhoa } \\
\text { Franklinia } \\
\text { Freziera } \\
\text { Gordonia } \\
\text { Killipiodendron } \\
\text { Laplacea } \\
\text { Paranneslea } \\
\text { Pyrenaria } \\
\text { Schima } \\
\text { Sladenia } \\
\text { Stuartia } \\
\text { Symplococarpon } \\
\text { Ternstroemia } \\
\text { Ternstroemiopsis } \\
\text { Tutcheria } \\
\text { Visnea }\end{array}$ & $\begin{array}{l}\text { Adinandra } \\
\text { Anneslea } \\
\text { Apterosperma } \\
\text { Archboldiodendron } \\
\text { Balthasaria } \\
\text { Camellia } \\
\text { Cleyera } \\
\text { Dankia } \\
\text { Eurya } \\
\text { Euryodendron } \\
\text { Ficalhoa } \\
\text { Freziera } \\
\text { Gordonia } \\
\text { Pyrenaria } \\
\text { Schima } \\
\text { Sladenia } \\
\text { Stewartia } \\
\text { Symplococarpon } \\
\text { Ternstroemia }\end{array}$ \\
\hline
\end{tabular}

monofilética del taxón.

Relaciones: es un género relacionado con Eurya y Cleyera.

Diversidad: género monotípico (E. excelsum Hung T. Chang).

Distribución: sur de China.

Referencia: Chang (1963).

19. Ficalhoa Hiern, J. Bot. 36: 329. 1898.

Se propone como posible autopomorfia la transición de poros apicales a aberturas longitudinales en las anteras de un mismo individuo.
Este taxón fue descrito por Hiern en 1898 como un género de Ericaceae, basado en la dehiscencia poricida de los anteras y las semillas pequeñas y numerosas que se producen en una cápsula loculicida. Recientemente, Robson (1961) incluyó a este género dentro de las Theaceae, aunque existen algunas características que no se presentan en los demás géneros de la familia, como la existencia de látex en la corteza y la inflorescencia dispuesta en cimas dicasiales. Sin embargo, por características del polen y de anatomía de madera se incluye en esta familia.

Relaciones: Robson (1961) ha sugerido una relación con Eurya, Cleyera y Freziera. 
Diversidad: género monotípico ( $F$. laurifolia Hiern). Distribución: África (de Uganda a Tanganika y Angola). Referencia: Robson (1961).

20. Sladenia Kurz, J. Bot. 11: 194. 1873.

La monofilia de este género puede definirse por sus filamentos cortos, redondeados y engrosados en la base y la dehiscencia de las anteras por poros apicales.

La ubicación taxonómica de Sladenia es incierta; ha sido incluido en las Dilleniaceae, las Linaceae y las Actinidaceae, e inclusive en una familia propia, Sladeniaceae. Gilg (1893 en: Kobuski 1951b) fue el primero en segregar al género de las Theaceae y ubicarlo en las Dilleniaceae junto con Actinidia, Clematoclethra y Saurauia. Gilg y Wedermann (1924 en: Kobuski 1951b) lo colocaron junto con los tres géneros anteriormente citados dentro de la familia Actinidiaceae; por su parte Hallier (1924) lo colocó dentro de las Linaceae. Metcalfe y Chalk (1950), con base en estudios anatómicos, lo consideraron como uno de los "géneros anómalos" de Theaceae. Estudios palinológicos (Kobuski 1951b) y anatómicos (Record 1942) apoyan su posición en las Theaceae; sin embargo, debido a que presenta una cima dicasial, un número menor de estambres (ca. 10) con anteras pilosas y filamentos cortos y ensanchados, Kobuski (1951b) lo ubica en una subtribu propia, Sladeniinae.

Relaciones: las relaciones de este género son inciertas, aunque se le ha relacionado con Ternstroemia y con Eurya.

Diversidad: género monotípico ( $S$. celastrifolia Kurz). Distribución: sureste de Asia (sur de China, Tailandia y Birmania).

Referencia: Kobuski (1951b).

\section{Géneros excluidos de Theaceae}

A continuación se presenta una lista de géneros que en alguna ocasión fuerom ubicados dentro de la familia Theaceare. Asimismo se indica en qué familia se acepta que están contenidos actualmente.

\section{Actinidia Lindl. (Actinidiaceae)}

Anthodiscus G. Mey. (Caryocaraceae)

Apatelia DC. = Saurauia Willd. (Actinidiaceae)

Archytaea Mart. (Bonnetiaceae sensu Takhtajan 1980)

Asteropeia Thouars (Asteropeiaceae sensu Takhtajan 1980).

Blumea G. Don = Saurauia Willd. (Actinidiaceae) Bonnetia Mart. (Bonnetiaceae sensu Takhtajan 1980)

Caraipa Aubl. (Clusiaceae sensu Stevens 1996ab) Caryocar L. (Caryocaraceae)
Cespedezia Ruiz \& Pav. (Ochnaceae) Clematoclethra Maxim. (Actinidiaceae) Cochlospermum Kunth (Bixaceae sensu Cronquist 1981; Cochlospermaceae sensu Takhtajan 1980) Dicaly $x$ Poir. $=$ Dicalix Lour. $=$ Symplocos Jacq. (Symplocaceae)

Euryanthe Cham. \& Schldl. = Amoreuxia Moç. \& Sessé (Cochlospermaceae)

Godoya Ruiz \& Pav. (Ochnaceae)

Haploclathra Benth. (Clusiaceae sensu Stevens 1996ab) Kielmeyera Mart. (Clusiaceae sensu Stevens 1996ab) Leucoxylon G. Don = Leucoxylum Blume $=$ Diospyros L. (Ebenaceae)

Mahurea Aubl. (Clusiaceae sensu Stevens 1996ab)

Marcgravia L. (Marcgraviaceae)

Marila Sw. (Clusiaceae sensu Stevens 1996ab)

Medusagyne Baker (Medusagynaceae sensu Takhtajan 1980)

Microsemma Labill. = Lethedon Spreng. (Thymeleaeceae)

Neotatea Maguire (Clusiaceae sensu Stevens 1996ab)

Norantea Aubl. (Marcgraviaceae)

Omphalocarpum P. Beauv. (Sapotaceae)

Pelliciera Planch. \& Triana (Pellicieraceae)

Pentamerista Maguire (Tetrameristaceae)

Pentaphylax Gardner \& Champ. (Pentaphylacaceae)

Ploiarium Korth. (Bonnetiaceae sensu Stevens 1996ab)

Poecilandra Tul. (Ochnaceae)

Poeciloneuron Bedd. (Clusiaceae)

Quiina Aubl. (Clusiaceae)

Ruyschia Jacq. (Marcgraviaceae)

Saurauia Willd. (Actinidiaceae)

Stachyurus Siebold \& Zucc. (Stachyuraceae sensu Cronquist 1981; Actinidiaceae sensu Takhtajan 1980) Symplocos Jacq. (Symplocaceae)

Tetramerista Miq. (Tetrameristaceae)

Tremanthera P. \& K. = Saurauia Willd. (Actinidiaceae)

Ventenatia P. Beauv. = Oncoba Forssk. (Flacourtiaceae)

\section{Discusión}

Los 20 géneros que se consideran en este trabajo como miembros de la familia Theaceae presentan una distribución tropical y subtropical, principalmente en Asia y América, con pocos elementos presentes en África. Un mayor porcentaje de géneros se concentra en el este de Asia y sus patrones de distribución coinciden con el trazo generalizado del Pacífico propuesto por Croizat (1964). Los pocos taxones con una distribución en África tienen mayor relación biogeográfica 
con los elementos de Asia y se orientan con una línea de base ubicada en el Océano Índico. Los análisis preliminares realizados hasta el momento (Luna y Weitzman en prep.) permiten tener elementos para sugerir que la familia es muy antigua y que tuvo una distribución en el pasado mucho más extensa, lo cual parece corroborarse por el hallazgo de fósiles en Europa (Gothan y Weyland 1954). Por otra parte, la presencia de varios de estos géneros en las Antillas refuerza la hipótesis de que tienen un origen pacífico.

Una vez definidos los elementos de la familia, es posible evaluar su conocimiento taxonómico con la información biogeográfica y, como menciona Croizat (1964), esto puede ser una fuente de evidencia o iluminación recíproca para entender mejor la historia natural de la familia, bajo una concepción que comprenda las relaciones filogenéticas y biogeográficas (históricas) de manera más robusta.

\section{Agradecimientos}

Agradecemos a la Dra. Anna L. Weitzman las numerosas revisiones al texto y varias de las ideas originales que se manejan en este artículo, incluyendo las numerosas sugerencias para delimitar a los géneros. Agradecemos al Dr. Rafael Lira y al Dr. Jorge Llorente la revisión crítica del manuscrito. Agradecemos al Dr. Peter Stevens de la Universidad de Harvard, el compartir con nosotros datos no publicados acerca de sus estudios en Clusiaceae y Bonnetiaceae. La primera autora agradece a la Fundación UNAM y a la DGAPA-UNAM los apoyos económicos recibidos para realizar estancias de trabajo en los herbarios de Estados Unidos e Inglaterra; este trabajo forma parte de los resultados de tales estancias.

\section{Literatura citada}

Airy-Shaw H.K. 1936. Notes on the genus Schima and on the classification of the Theaceae-Camellioideae. Bulletin of miscellaneous information 1936:496-499.

Airy-Shaw H.K. 1939. Symplococarpon hintonii (Bullock) AiryShaw. En: A.W. Hill (Ed.). Hooker's Icones Plantarum 34: pl. 3342. London.

Baillon H.E. 1873. XXX. Ternstroemiacées. En: Histoire des Plantes. Tome IV. L. Hachette et Cie. Paris, Pp. 227-264.

Barker W.R. 1980. Taxonomic revisions in Theaceae in Papuasia. I. Gordonia, Ternstroemia, Adinandra, and Archboldiodendron. Brunonia 3:1-60.

Bentham G. 1861. Notes on Ternstroemiaceae. Journal of the proceedings of the Linnean Society, Botany 5:53-65.

Bentham G, Hooker J.D. 1862. Genera Plantarum. Vol. I: 177188. London.

Bessey C.E. 1915. The phylogenetic taxonomy of flowering plants. Annals of the Missouri Botanical Garden 2:109-164.
Bloembergen S. 1952. A critical study in the complex polymorphous genus Schima (Theaceae). Reinwardtia 2:133-183.

Blume C.L. 1825. Ternstroemiaceae. En: Bijdragen tot de Flora van Nederlandsch Indië. 1ste Stuk. ter Lands Drukkerij, Batavia. Pp. 124-131.

Brummitt R.K. (comp). 1992. Vascular plant families and genera. Royal Botanic Gardens, Kew. Great Britain. 804 p.

Burkill I.H. 1917. Gordonia. Journal of the straits branch of the royal asiatic society 76:133-159.

Cambessèdes J. 1828. Mémoire sur les familles des Ternstroemiacées et des Guttifères. Mémoires du Museum d'Histoire Naturelle Paris 16:369-416.

Candolle A.P. de. 1822. Mémoire sur la Famille des Ternstroemiacées, et en particulier sur le genre Saurauja. Mémoires de la société de physique et d'histoire naturelle de Genève 1 (2):393-430.

Candolle A.P. de. 1824. Ternstroemiaceae. En: Prodromus systematis naturalis regni vegetabilis. Vol. I. Treuttel et Wurtz, Paris. Pp 523-530.

Chang H.T. 1963. Euryodendron, a new genus of Theaceae. Acta Scientiarium Naturalium Universitatis Sunyatseni 1963 (4):129-131.

Chang H.T. 1976. Apterosperma - Cenus Novum Theacearum. Acta Scientiarium Naturalium Universitatis Sunyatseni 1976 (2):89-92.

Chang H.T. 1981. A taxonomy of the genus Camellia. Journal of Sun Yatsen University 4:1-180.

Chang H.T. y Bartholomew B. 1984. Camellias. Timber Press. Portland, Oregon. Pp 211.

Choisy J.D. 1855. Mémoire sur les familles des Ternstroemiacées et Camelliacées. Mémoires de la société de physique et d'histoire naturelle de Genève 14:91-186.

Croizat, L. 1964. Space, time and form: the biological synthesis. Publicado por el autor. Caracas, Venezuela.

Cronquist A. 1981. An integrated system of Classification of Flowering Plants. Columbia University Press. New York. Pp 1262.

Dahlgren R.M.T. 1983. General aspects of angiosperm evolution and macrosystematics. Nordic Journal of Botany 3 : 119-149.

Dalla Torre C.W. von y Harms H.A.T. 1963. Genera Siphonogamarum. Lipsiae. Pp. 317-318.

Don D. 1825. Prodromus Flora Nepalensis. J. Gale, London. pp. 224-225.

Don G. 1831. A general history of the dichlamydeous plants. Vol. I. Thalamiflorae. London. Pp. 563-579.

Endlicher S.L. 1836-1840, 1843. Ordo Ternstroëmiaceae. En: Generum Plantarum. Fr. Beck, Wien. suppl. II p. 81, suppl. III p. 1017-1924.

Engler A. 1908. Theaceae. En: Engler A, Prantl E. Die natürlichen Pflazenfamilien. Leipzig. Pp. 226-227.

Gagnepain F. Dankia. 1939. En: Humbert H. Supplément a la flore générale de l'Indo-Chine. Tome Premier, p. 198-199, 202.

Goldberg A. 1986. Classification, Evolution and Phylogeny of the families of Dicotyledons. Smithsonian Contributions to Botany No. 58. Smithsonian Institution Press. Washington.

Gothan W., Weyland H. 1954. Lehrbuch der Paläobotanik. Akademie-Verlag. Berlin.

Greuter W (Chairman editor). 1994. International Code of Botanical Nomenclature (Tokyo Code). Koeltz Scientific Books. Königstein, Germany. 
Grote P.J, Dilcher D.L. 1989. Investigations of angiosperms from the Eocene of North America: a new genus of Theaceae based on fruit and seed remains. Botanical Gazette 150:190-206.

Hallier H. 1916. Marcgraviaceae. En: Beiträge zur Flora von Borneo. Beihefte zum Botanischen Centralblatt 34 (2): 35-40.

Hallier H. 1924. Ueber die Lennoeen eine zu Linne's Bicornes verirrte sippe der Borraginaceen. Beihefte zum Botanischen Centralblatt 40 (2):1-19.

Heywood V.H. (Ed). 1979. Flowering Plants of the World. Oxford University Press. Oxford. 335 p.

Hiern W.P. 1898. A new genus of Ericaceae from Angola. Journal of Botany 36:329-330.

Hutchinson J. 1973. Families of flowering plants. Third Edition. Oxford University Press. Great Britain. p. 366-372.

Jussieu A. L. de. 1789. Genera Plantarum. Tomus XXXV. Herissant \& Barois, Paris. p. 262.

Keng H. 1962. Comparative morphological studies in Theaceae. University of California publications in Botany 33: 269384.

Keng H. 1972. Two new theaceous plants from Malaysia and a proposal to reduce Tutcheria to a synonym of Pyrenaria. Gardens' Bulletin Singapore 26 (1):127-135.

Keng H. 1980a. On the unification of Laplacea and Gordonia (Theaceae). Gardens' Bulletin Singapore 33 (2):303-311.

Keng H. 1980b. The genus Pyrenaria (Theaceae) in Malesia (Flora Malesianae Precusores LVIII, Part One). Gardens' Bulletin Singapore 33 (2):264-289.

Keng H. 1984. Florae Malesianae Precursores - LVIII, Part Two. The genus Gordonia (Theaceae) in Malesia. Gardens' Bulletin Singapore 37 (1):1-47.

Kobuski C.E. 1935. Studies in the Theaceae. I. Eurya subgen. Ternstroemiopsis. Journal of the Arnold Arboretum 16: 347-353.

Kobuski C.E. 1937. Studies in the Theaceae. II. Cleyera. Journal of the Arnold Arboretum 18:118-129.

Kobuski C.E. 1938. Studies in the Theaceae. III. Eurya subgenera Euryodes and Penteurya. Annals of the Missouri Botanical Garden 25:299-359.

Kobuski C.E. 1940. Studies in the Theaceae. V. The Theaceae of New Guinea. Journal of the Arnold Arboretum 21: 134-162.

Kobuski, C.E. 1941a. Studies in the Theaceae. VI. The genus Symplococarpon Airy-Shaw. Journal of the Arnold Arboretum 22:188-196.

Kobuski C.E. 1941b. Studies in the Theaceae. VII. The American species of the genus Cleyera. Journal of the Arnold Arboretum 22:395-416.

Kobuski C.E. 1941c. Studies in the Theaceae. VIII. A synopsis of the genus Freziera. Journal of the Arnold Arboretum 22:457-496.

Kobuski C.E. 1942. Studies in the Theaceae. XII. Notes on the South American species of Ternstroemia. Journal of the Arnold Arboretum 23:298-343.

Kobuski, C.E. 1943. Studies in the Theaceae. XIV. Notes on the West Indian species of Ternstroemia. Journal of the Arnold Arboretum 24:60-76.

Kobuski C.E. 1947. Studies in the Theaceae. XV. A review of the genus Adinandra. Journal of the Arnold Arboretum 28:1-98.
Kobuski C.E. 1949. Studies in the Theaceae. XVIII. The West Indian species of Laplacea. Journal of the Arnold Arboretum 30:166-186.

Kobuski C.E. 1950. Studies in the Theaceae. XX. Notes on the South and Central American species of Laplacea. Journal of the Arnold Arboretum 31:405-429.

Kobuski C.E. 1951a. Studies in the Theaceae. XXI. The species of Theaceae indigenous to the United States. Journal of the Arnold Arboretum 32:123-138.

Kobuski C.E. 1951b. Studies in the Theaceae. XXIV. The genus Sladenia. Journal of the Amold Arboretum 32:403-409.

Kobuski C.E. 1952a. Studies in the Theaceae. XXV. The genus Anneslea. Journal of the Arnold Arboretum 33:79-90.

Kobuski C.E. 1952b. Studies in the Theaceae. XXVI. The genus Visnea. Journal of the Arnold Arboretum 33:188-191.

Kobuski C.E. 1956. Studies in the Theaceae. XXVIII. Melchiora, a new genus in Africa. Journal of the Arnold Arboretum 37:153-159.

Kobuski C.E. 1957. Studies in the Theaceae. XXIX. Further studies in the genus Melchiora. Journal of the Arnold Arboretum 38:199-205.

Kobuski C.E. 1961a. Studies in the Theaceae. XXX. The African species of Ternstroemia. Journal of the Arnold Arboretum 42:81-86.

Kobuski C.E. 1961b. Studies in the Theaceae. XXXII. A review of the genus Ternstroemia in the Philippine Islands. Journal of the Arnold Arboretum 42:263-275.

Kunth C.S. 1822. Malvaceae, Büttneriaceae, Tiliaceae. En: Humboldt A, Bonpland A. Nova Genera et Species Plantarum.

Lawrence G.M. 1951. Taxonomy of vascular plants. The Macmillan Co. New York. 823 p.

Ling L.K. 1966. A revision of genus Eurya in China. Acta Phytotaxonomica Sinica 11 (3):263-340.

Linneo C. von. 1753. Species Plantarum. 1a. Ed. Stockholm. Pp. 515, 698 y 783.

Luna I., Almeida L., Villers L., Lorenzo L. 1988. Reconocimiento florístico y consideraciones fitogeográficas del bosque mesófilo de montaña de Teocelo, Veracruz. Boletín de la Sociedad Botánica de México 48:35-63.

Luna I., Almeida L., Llorente J. 1989. Florística y aspectos fitogeográficos del bosque mesófilo de montaña de las cañadas de Ocuilan, estados de Morelos y México. Anales del Instituto de Biología, UNAM, Serie Botánica 59 (1):63-87.

Luna I., Ocegueda S., Alcántara O. 1994. Florística y notas biogeográficas del bosque mesófilo de montaña del municipio de Tlanchinol, Hidalgo, México. Anales del Instituto de Biología UNAM, Serie Botánica 65:31-62.

Llorente J., Espinosa D. 1992. Una síntesis de las controversias en la biogeografía histórica contemporánea. Ciencia 42 (3):295-312.

Maguire B., Zeeuw C. de, Huang Y-C., Clare C.C. Jr. 1972. Bonnetiaceae. En: The Botany of the Guayana Highland Part IX. Memoirs of the New York Botanical Garden 23:131-165.

Melchior H. 1925. Theaceae. En: Engler A, Prantl E. Die Natürlichen Pflazenfamilien, 2d. ed., Leipzig. 21:109-154.

Metcalfe C.R., Chalk L (Eds). 1950. Anatomy of the dicotyledons; leaves, stem, and wood in relation to Taxonomy, with notes on economic uses. 2 vols. Clarendon Press. Oxford.

Metcalfe C.R., Chalk L (Eds). 1972. 50. Theaceae y 52. Bonnetiaceae. En: Anatomy of the Dicotyledons. 2a. Ed. 
Oxford University Press. Pp. 181-191 y 193-195.

Mirbel C.F.E. de. 1813. Notes pour servir à $\mathbf{l}^{\prime}$ Histoire Naturelle de la famille des Orangers de MA-L de Jussieu. Nouveau Bulletin des Sciences par la société Philomatique de Paris 3:376-383.

Nelson G., Platnick N.I. 1981. Systematics and Biogeography, Cladistics and Vicariance. Columbia University Press. New York. 567 p.

Phamhoang H. 1991. Theaceae. En: An Illustrated Flora of Vietnam. Cayco Vietnam. Pp. 511-539.

Record S.J. 1942. American woods of the family Theaceae. Tropical woods 70:23-33.

Robson K.B. 1961. The taxonomic position of Ficalhoa laurifolia Hiern. Comptes Rendus de la IVe Réunion Aetfat. Lisboa. p. 299-308.

Rzedowski J. 1991. Diversidad y orígenes de la flora fanerogámica de México. Acta Botánica Mexicana 14:3-21.

Schofield E.K. 1968. Petiole anatomy of the Guttiferae and related families. Memoirs of the New York Botanical Garden 18 (1):1-55.

Seetharam Y.N. 1985. Clusiaceae: Palynology and Systematics. Institut Français de Pondichéry. Travaux de la Section Scientifique et Technique. Tome XXI. 80 p. y láms.

Spach E. 1835. Les Ternstrémiacées - Ternstroemiaceae. En: Histoire naturelle des végétaux. Vol. IV. Paris. pp. 58-99.

Spongberg S.A. 1974. A review of deciduous-leaved species of Stewartia (Theaceae). Journal of the Arnold Arboretum 55:182-214.

Stevens P. 1996a. A descriptive data matrix of the Clusiaceae. Unpubl.

Stevens P. 1996b. Clusiaceae. En: Kubitzki K. The genera of vascular plants. Unpubl.
Szyszylowicz I. von. 1893. Theaceae (Ternstroemiaceae). En: Engler A, Prantl E. Die natürlichen Pflanzenfamilien, $3^{6}$ : 175-192. Leipzig.

Takhtajan A. 1980. Outline of the classification of flowering plants (Magnoliophyta). Botanical Review 46:225-359.

Thorne R.F. 1992. Classification and geography of the flowering plants. Botanical Review 58 (3):225-350.

Thunberg C.P. 1783. Nova Genera Plantarum. Partem primam. Joh Edman, Upsaliae. Pp. 67-70.

Tsou Ch. 1995. Embryology of Theaceae - anther and ovule development of Adinandra, Cleyera and Eurya. Journal of Plant Research 108:77-86.

Tulasne L.R. 1847. Plantes nouvelles de la Colombie. Annales des Sciences Naturelles; botanique, Paris 3, 8: 326-346.

Urban I. 1896. Ternstroemiaceae (sensu ampliore). En: Engler A. Systematik, Pflanzengeschichte und Pflanzengeographie. Engler's Botanische Jahrburher 21. Leipzig. p. 514-551.

Verdcourt B. 1969. Melchiora Kobuski and Melchioria Penzig \& Saccardo. Kew Bulletin 23:469-470.

Weitzman A.L. 1987. Systematics of Freziera Willd. (Theaceae). $\mathrm{Ph}$. D. Dissertation. Harvard University. Cambridge, Massachusetts. 327 p.

Weitzman A.L. 1995. Diversity of Theaceae and Bonnetiaceae in the Montane Neotropics. En: Churchill SP et al. Biodiversity and Conservation of Neotropical Montane Forests. The New York Botanical Garden. pp. 365-375. (en prensa).

Yan S. Z. 1981. On the Chinese genera Stewartia Linn. and Hartia Dunn. Acta Phytotaxonomica Sinica 19 (4):462-471.

Ye C.X. 1982. A taxonomy of the genera Stewartia and Hartia. Acta Scientiarium Naturalium Universitatis Sunyatseni 1982 (4):108-116. 